\title{
In Situ Lift-Out for Coordinated Structure-Electron Transport and Structure-Isotope Studies
}

\author{
R.M. Stroud*, T.J. Zega* and M.L. Taheri* \\ * Materials and Sensors Branch, Naval Research Laboratory, 4555 Overlook Avenue, Washington, \\ DC 20375
}

The scientific value of coordinated studies of the same material greatly exceeds that of individual analyses. For example, the analysis of meteoritic material frequently requires isotope measurements to identify the cosmic origin of the material, as well as structural analysis to determine the formation and processing conditions. Together, structure and isotope data on a single sub-micron grain can allow the identification of the particular class of star in which the grain condensed, and determination of whether the condensation process in that particular star occurred in thermodynamic equilibrium [1]. Thus, coordinated nanoscale analyses are essential for advancing astrophysical models of stellar evolution. Similarly, our understanding of the electrical properties of nanostructures can benefit from the ability to carry out both transmission electron microscopy (TEM) studies and transport measurements on an individual nanostructure because resistance properties can be directly related to microstructure [2].

In situ lift-out using a micromanipulator probe that also serves as a TEM sample support (hereafter microtweezer) is a highly adaptable technique for enabling the coordinated analyses. We use the Ascend Extreme Access lift-out tool, mounted on a FEI Nova 600 focused ion beam - scanning electron microscope, to extract samples ranging from sub-micron dust grains that pre-date the solar system to individual GaN nanowires. Subsequent analyses of the meteoritic grains can include TEM, isotopic characterization by secondary ion mass spectroscopy (SIMS), scanning electron microscopy, as well as synchrotron-based analyses such as x-ray absorption near-edge spectroscopy (XANES). For nanowires, typical analyses are analytical TEM combined with in situ point-contact I-V measurements, obtained with a Nanofactory STM-TEM system.

Figure 1 shows a schematic of the microtweezer lift-out probe and sample support (a), and example images of the lift-out process (b). Molybdenum and copper microtweezers are available from Ascend under the product name End-Effector ${ }^{\mathrm{TM}}$. The final dimensions of the tweezer fork can be customized to the particular sample requirements prior to lift-out. This approach works well for the standard sample geometry, and also for sectioning sub-micron particles and nanowires. After liftout, the microtweezer can be folded so that the sample is held in the center of a 3-mm u-shape, compatible with most TEM samples holders, or left unfolded depending the sample geometry requirements of the desired analysis instruments. Our presentation will include more example applications, as well detailed results from the coordinated TEM- SIMS analyses of primitive extraterrestrial materials (e.g., Fig.2) and GaN nanowire microstructure-transport studies [3].

References

[1] R.M. Stroud et al., Science 305 (2004) 1455.

[2] M.L. Taheri, et al., this volume. 
[3] TJZ and MLT gratefully acknowledge support from the NRL/NCR Postdoctoral Fellowship program. We thank Jorgen Rasmussen and Glen Geller of Ascend for assistance with the Extreme Access system.
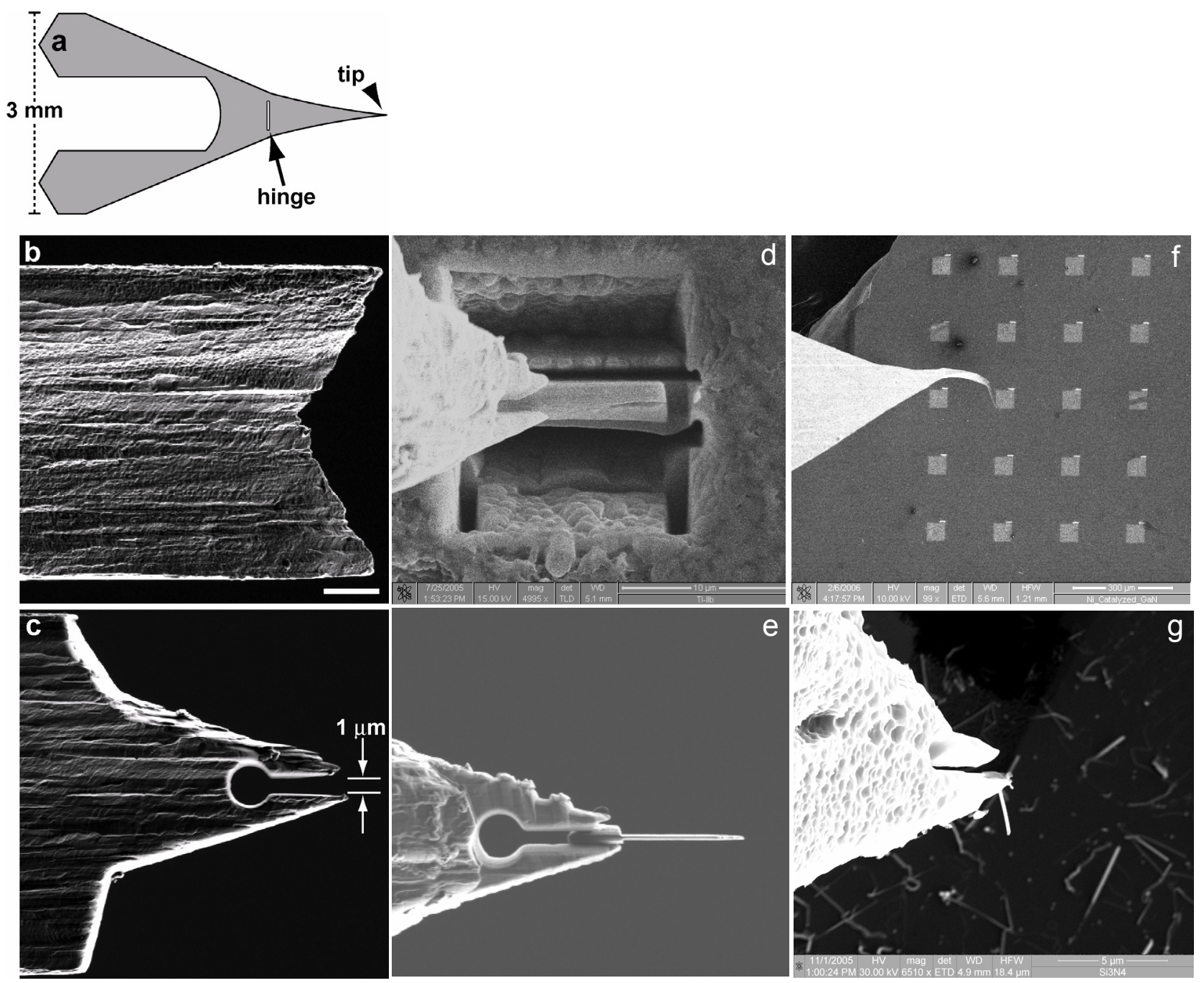

FIG 1. An overview of the microtweezer approach to in situ lift-out. A. Schematic of the microtweezer sample support. B. Secondary-electron image of an unpatterned microtweezer probe tip. C. FIB-patterned microtweezer fork. D. Extraction of a sample with the microtweezer. E. An extracted sample thinned to electron transparency. F. A microtweezer approaching a patterned silicon substrate with nanowires. G. Extraction of an individual nanowire.

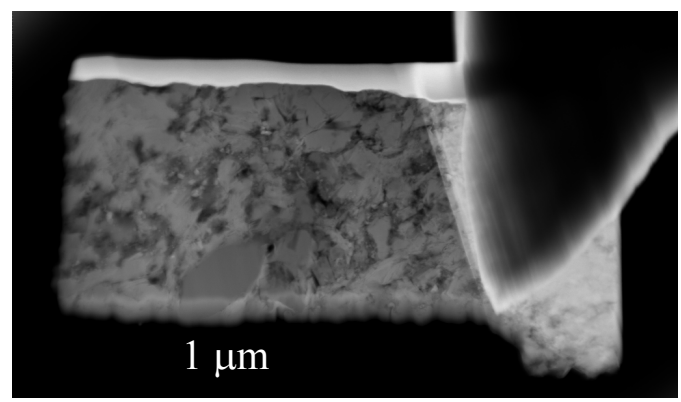

FIG 2. Z-contrast image of a microtweezer-supported meteorite section. 